\title{
Red Blood Cells Mimics Production in a Flow Focus Device with Rectangular Cross Section
}

\author{
A. I. Moreira ${ }^{1}$, J. Carneiro' ${ }^{1}$, J. B. L. M. Campos' ${ }^{1}$ J. M. Miranda ${ }^{1}$ \\ ${ }^{1}$ CEFT, Transport Phenomena Research Center, Chemical Engineering Department \\ Faculty of Engineering, University of Porto, Rua Roberto Frias s/n, 4050-465 Porto, Portugal \\ aidm@fe.up.pt, jcarneiro@fe.up.pt; jmc@fe.up.pt; jmiranda@fe.up.pt
}

\begin{abstract}
The conditions to produce hydrogel Red Blood Cells (RBC) mimics were studied by experimental methods. Hydrogel microparticles were produced in a microfluidic device. Several operating conditions (the continuous and dispersed phase flow rates) were studied and optimize to produce particles with sizes and mechanical properties similar to the ones of RBCs. Particles with approximately $50 \mu \mathrm{m}$ can be produced with this technique in a channel if $100 \times 50 \mu \mathrm{m}^{2}$. To produce microparticles with a size similar to RBCs, a channel with lower dimensions is required.
\end{abstract}

Keywords: gelatin, microdroplets, droplet microfluidics, microchannels, microsystems, hydrogels

\section{Introduction}

Sometimes the experiments to test medical devices and drug delivery system need living cells and tissues. The use of these biological materials has high costs and present ethical concerns. However, not all the tests require living systems, sometimes it is possible to use fluids that can mimic the rheological and mechanical behavior of the biological fluids. Among all the biological fluids, blood is one of the fluids that presents a great interest to be mimicked.

Blood is a suspension composed by red blood cells (RBCs), white cells, platelets and plasma. RBCs are the cells present in greater quantity in blood, they present a biconcave disk shape, a diameter of $\sim 8 \mu \mathrm{m}$ and a thickness of $\sim 2 \mu \mathrm{m}[1]$. There is the possibility to obtain microparticles with the same properties that the RBCs.

Hydrogels are a class of materials that can be used to mimic the RBCs. They present good surface properties and are biocompatible [2]. These characteristics make them attractive for drug delivery, cell encapsulation, tissue engineering and other biomedical applications [2]. Hydrogels are obtained by crosslinking from monomers in aqueous solutions, which can be induced by different changes in conditions, such as changes in temperature, $\mathrm{pH}$ or addition of an initiator.

Droplet microfluidics present several advantages to produce hydrogel microparticles, such as the lower reactant consumption and the ability to control the size and shape of the microparticles. With this technique, it is possible to obtain highly monodisperse microparticles [3].

Gelatin is a natural hydrogel suitable for medical and pharmaceutical applications due to its biocompatibility, biodegradability, non-toxicity and easy removal in physiological environmental [4]. Gelatin is thermoreversible and becomes a gel when the temperature decreases bellow $25^{\circ} \mathrm{C}$ (for a solution of $1 \%(\mathrm{w} / \mathrm{v})$ ) [4].

The aim of this work is the production of gelatin microdroplets that can mimic the RBCs using droplet microfluidics. Experiments are conducted to optimize the conditions to produce these microparticles in a flow focusing device in which the main channel has a rectangular cross section.

\section{Methods}

\subsection{Device Fabrication}

The microchannels were fabricated in PDMS by soft lithography using a SU-8 molds, following our previous work [5]. The microchannel used has two inlets, one for the continuous phase and another for the dispersed phase, and one outlet. The microchannel used has a rectangular section with a width of $100 \mu \mathrm{m}$ and a height of $50 \mu \mathrm{m}-$ Figure 1 . 
a)

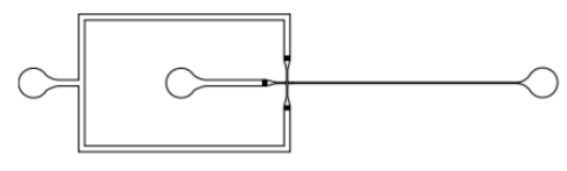

C)

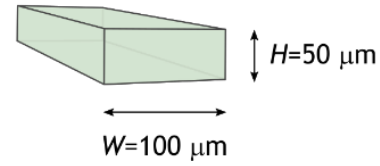

b)

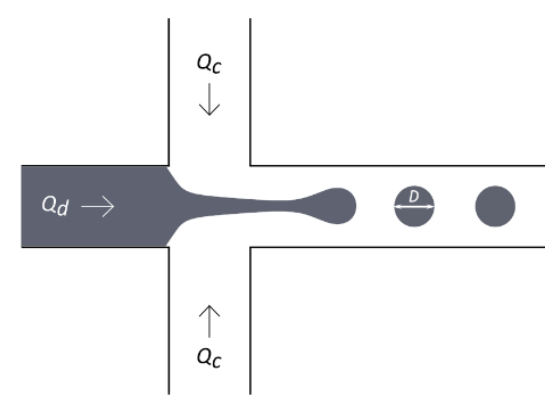

Figure 1 Scheme of the microchannel used to produce de gelatin microdroplets: a) full view of the microchannel; b) scheme of the intersection of the two phases in the microchannel; c) $3 \mathrm{D}$ view of the microchannel section $W=100 \mu \mathrm{m}$ and $H=50 \mu \mathrm{m}$.

\subsection{Fluid Properties}

Gelatin from porcine skin was used as the dispersed phase, light mineral oil was used as the continuous phase and Span 80 was used as surfactant. All the materials were from Sigma Aldrich. The fluid properties are presented in Table 1.

Table 1 Fluid properties.

\begin{tabular}{|c|c|c|c|}
\hline Fluid & Viscosity (Pa.s) & Density $\left(\mathrm{kg} / \mathrm{m}^{3}\right)$ & Interfacial tension $(\mathrm{N} / \mathrm{m})$ \\
\hline 5 wt\% gelatin in distilled water & 0.0050 & 1008.9 & \multirow{2}{*}{0.0038} \\
\hline Mineral oil + 3 wt\% Span 80 & 0.0151 & 854.1 & \\
\hline
\end{tabular}

\subsection{Flow Visualization and Characterization}

A high-speed camera (FASTCAM mini UX100, Photron) mounted on an inverted epifluorescence microscope (DMI $5000 \mathrm{M}$, Leica Microsystems Gmbh) was used to capture the microdroplet formation images. A syringe pump connected to the computer was used to inject the continuous and dispersed phases in the microchannel with controlled flow rates. The diameters of the microdroplets were measured using the high-speed camera software, Photron FASTCAM Viewer 4.

\section{Results}

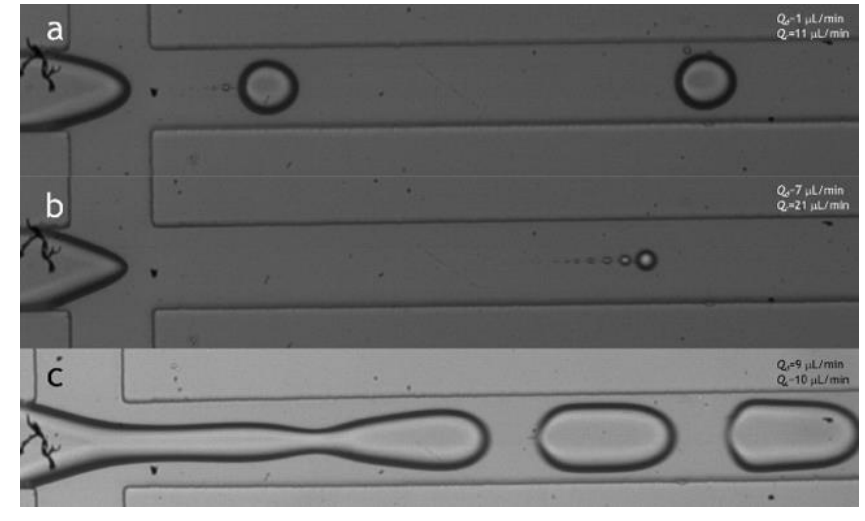

Figure 2 Regimes found for the viscosity ratio of 0.33 : a) dripping $\left.\left(Q_{d}=1 \mu \mathrm{L} / \mathrm{min} ; Q_{c}=11 \mu \mathrm{L} / \mathrm{min}\right) ; \mathrm{b}\right)$ tip muti-breaking $\left.\left(Q_{d}=7 \mu \mathrm{L} / \mathrm{min} ; Q_{c}=21 \mu \mathrm{L} / \mathrm{min}\right) ; \mathrm{c}\right)$ jetting $\left(Q_{d}=9 \mu \mathrm{L} / \mathrm{min}\right.$; $Q_{c}=10 \mu \mathrm{L} / \mathrm{min}$. (width of the channel $=100 \mu \mathrm{m}$ ).

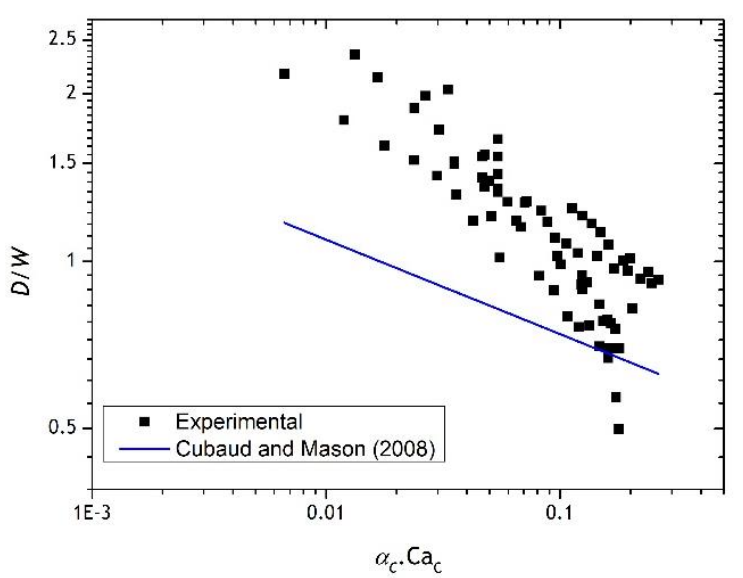

Figure $3 \mathrm{D} / \mathrm{W}$ vs $\alpha_{c}$. $C a_{c}$ : comparison between the data of this work and Cubaud and Mason [3] work. 
Several flow rates of the continuous and dispersed phase were tested in the microchannel for a viscosity ratio $\left(\mu_{\mathrm{d}} / \mu_{\mathrm{c}}\right)$ of 0.33. Dripping, jetting, tubing, and a regime similar to tip multi-breaking [6] were found - Figure 2. The variation of the microdroplets size was studied for the dripping and jetting regime. The smallest droplet was found for dripping $(49.82 \mu \mathrm{m}-$ $\left.Q_{d}=3 \mu \mathrm{L} / \mathrm{min} ; Q_{c}=16 \mu \mathrm{L} / \mathrm{min}\right)$. For the jetting the smallest droplet found was $120.35 \mu \mathrm{m}\left(Q_{d}=7 \mu \mathrm{L} / \mathrm{min} ; Q_{c}=11 \mu \mathrm{L} / \mathrm{min}\right)$.

We compared our dripping results with Cubaud and Mason [3] results. We concluded that there is a large deviation from the Cubaud and Mason [3] results - Figure 3. This large deviation between the values shows that the model of prediction created by this author, does not apply to fluids with a low viscosity ratio. Cubaud and Mason [3] used fluids with viscosity ratio of 264 to obtain these results, while this work focus on a viscosity ratio of 0.33 .

\section{Conclusion}

A microchannel with $100 \times 50 \mu \mathrm{m}$ was used to produce gelatin microdroplets. Four regimes were identified - dripping, jetting, tip multi-breaking and tubing. The results obtained for dripping were compared with the results of Cubaud and Mason [3]. A very marked difference was found between the results, which demonstrates that the viscosity ratio of fluids has on droplet formation. A more in-depth study for fluids with a low viscosity ratio should be carried out, as the existing studies are for high viscosity ratios or channels quite different from the one used.

\section{Acknowledgements}

This work was funded by FEDER funds through COMPETE2020 - Operational Programme for Competitiveness Factors (POCI) and National Funds (PIDDAC) through FCT (Fundação para a Ciência e a Tecnologia) under project POCI01-0145-FEDER-016861-PTDC/QEQ-FTT/4287/2014 and Transport Phenomena Research Center - CEFT base funding $\mathrm{UIDB} / 00532 / 2020$.

\section{References}

[1] G. Tomaiuolo, "Biomechanical properties of red blood cells in health and disease towards microfluidics," Biomicrofluidics, vol. 8, no. 5, p. 051501, 2014, doi: 10.1063/1.4895755.

[2] A. Moreira, J. Carneiro, J. B. L. M. Campos, and J. M. Miranda, "Production of hydrogel microparticles in microfluidic devices: a review," Microfluidics and Nanofluidics, vol. 25, no. 2, 2021, doi: 10.1007/s10404-020-02413-8.

[3] T. Cubaud and T. G. Mason, "Capillary threads and viscous droplets in square microchannels," Physics of Fluids, vol. 20, no. 5, p. 053302, 2008, doi: 10.1063/1.2911716.

[4] C.-H. Yeh, K.-R. Chen, and Y.-C. Lin, "Developing heatable microfluidic chip to generate gelatin emulsions and microcapsules," Microfluidics and Nanofluidics, journal article vol. 15, no. 6, pp. 775-784, December 01 2013, doi: 10.1007/s10404-013-1193-X.

[5] J. Carneiro, E. Doutel, J. B. L. M. Campos, and J. M. Miranda, "PDMS droplet formation and characterization by hydrodynamic flow focusing technique in a PDMS square microchannel," Journal of Micromechanics and Microengineering, vol. 26, no. 10, p. 105013, 2016. [Online]. Available: http://stacks.iop.org/0960$1317 / 26 / \mathrm{i}=10 / \mathrm{a}=105013$.

[6] P. Zhu, T. Kong, Z. Kang, X. Tian, and L. Wang, "Tip-multi-breaking in Capillary Microfluidic Devices," Scientific Reports, vol. 5, no. 1, p. 11102, 2015/06/16 2015, doi: 10.1038/srep11102. 